\title{
A Survey on Face Recognition Techniques for Undersampled Data
}

\author{
Chikkala V S N Pavan Eswar Kumar' ${ }^{1}$, Dr. S. Phani Kumar² \\ ${ }^{1}$ M.Tech (CST), Department of Computer Science and Engineering, GITAM School of Technology, Hyderabad-502329, India \\ ${ }^{2}$ M.Tech, Ph.D., Professor \& HOD, Department of Computer Science and Engineering, GITAM School of Technology, Hyderabad-502329, \\ India
}

\begin{abstract}
With the increase of the Security threats around the world, it is very difficult to find a person whether he is an authorized one or not. Due to an increase of the Globalization and digitization around the world, the Face Recognition technology gains a lot of importance for identification purpose. These techniques are mainly focused on the authentication and authorization aspects and these methodologies will help us to locate any person by capturing and comparing those from the images that are present in the database, Even the disguised ones also. In this Survey paper, different types of Undersampled Face Recognition methods are analyzed.
\end{abstract}

Keywords: Undersampled Face Recognition, Authentication, and Authorization

\section{Introduction}

Humans typically use faces to acknowledge people however in today's data world pc primarily based recognition is needed. Face recognition has been a full of life analysis topic inside the Digital world. Various traditional approaches in the recognition such as are Correlation[3], Eigenfaces[4], and Sparse representation[13] since it is challenging to recognize face images with illumination and expression variations as well as corruptions due to occlusion or disguise. A typical solution is to collect a sufficient amount of training data in advance so that the above intraclass variations can be properly handled. However, in practice data collection in advance is impossible.

Existing solutions to undersampled face recognition can be typically divided into two categories: patch-based methods and generic learning [6] from external data. For achieving recognition in patch-based methods, one can extract discriminative information from patches collected by different images or integrating the corresponding results. In patch methods, the information for finding a person is limited, whereas in generic learning methods the information is extracted from external data via dictionary learning methodologies [11].

\section{a) How we can define Face Recognition}

A facial recognition is a computer-based real-time application capable of identifying or verifying a person from real-time sources either from an image or a video, which are used in security mechanisms by comparing the facial features from the image and a facial database. These Biometric mechanisms uses an automatic strategies of supportive or recognizing the identity of the living person on the idea of some physiological characteristics like fingerprints, countenance or eye iris for recognition.

\section{b) Major applications of Face Recognition}

Some of the major applications in which face recognition techniques are extensively used are:

1) Face Identification

2) Security

\section{3) Access control}

4) Surveillance

Let us consider an example, In military applications, Data confidentiality and integrity are more important for the security of any nation. Face recognition techniques are helpful to spot and verify whether or not the user is a certified one or to not access that information. during this kind of situation, the authorization can rely on face-based or eye-based identification. First, it will verify with the information and user image with the database which is present in the server side, then that particular user is an authorized one and will allow to access the data if he is an authenticated one.

In Medical field, Automation Face recognition techniques are used in which the necessary patient details are created and uploaded to the database if the patient is visiting the hospital for the first time. With the help of these techniques whenever the patient visits again, the doctor can check the patient previous health conditions to the present situation and will give appropriate medicine.

\section{c) Three basic approaches for Face Recognition}

Different approaches of face recognition are classified into three groups based on face representation. They are

a) Holistic approach

b) Feature-based approach

c) Hybrid approach

\section{Holistic Approach}

Holistic Approach is an appearance-based approach which uses holistic texture features and is applied to either wholeface or specific regions in a face image. This methodology is predicated on principal part analysis (PCA)[17] techniques which will be wont to scale back the spatiality of the info by holding the characteristics of the dataset. Subspace analysis is done by projecting an image into a lower dimensional subspace formed with the help of training face images and after that recognition is performed by measuring the distance between known images and the image to be recognized. Principal element Analysis (PCA)[17], Linear Discriminant 


\section{International Journal of Science and Research (IJSR) \\ ISSN (Online): 2319-7064}

Index Copernicus Value (2015): 78.96 | Impact Factor (2015): 6.391

Analysis (LDA)[17] are two most outlined algorithms often used within the face recognition.

\section{Feature-based Approach}

The feature-based approach uses geometric facial features like mouth, eyes, brows, cheeks, etc. and applies geometric relationships between them. Pure geometry, dynamic link architecture, and hidden Markov model methods belong to this category. The drawbacks of this method ar that it cannot restrained the amendment in element values, if the image is roofed with glasses, mask etc..or disguised.

\section{Hybrid Approach}

The hybrid approach is a combination of both appearances based and feature based approaches. Among the face recognition approaches, appearance-based approaches have been successfully developed and tested in many applications for face recognition. The above approaches also utilize the pixel intensity or intensity-derived features.

\section{Related Work}

In the last previous years Researches have shown interest in the various Face Recognition techniques and developed various efficient algorithms. Some noticeable add the world of face recognition is as follows:

In [2] T. Ahonen, A. Hadid and M. Pietikainen has proposed a sharpness metric to segment an image into blurred and not blurred parts and to separate in-band and out of band interferences of pixels, we used sharpness matrix based on Local Binary Pattern(LBP) and Robust segmentation algorithm.

In [3] Marios Kyperountas, Anastasios Tefas, Ioannis Pitas has proposed a methodology in multiple steps. At each clustering step, the test and training faces are projected to a Discriminant face and the data that is projected onto space are partitioned into clusters using the k-means algorithm. The training data set clusters are selected and compared with the test face, repeat the same process until the last cluster is compared with the test face then, that test face is matched if the distance is minimum.

In [4] M. Turk and A. Pentland has proposed a methodology in which the face images are projected onto feature space. The image is outlined within the kind of eigenvectors and every eigenvector may be a blurred image with low quality. Using these eigenvectors one can compare with the image present in the training dataset for better recognition purpose.

In [5] Peter N. Belhumeur, Joao P. Hespanha, and David J. Kriegman have proposed a methodology in which the face recognition can be possible even the image is subjected severe under varying lighting conditions and facial expressions.

In [6] Y. Su, S. Shan, X. Chen and W. Gao has proposed a Coupled Linear Representation (CLR) algorithm based on Adaptive Generic Learning (AGL) method, in which it adapts a generic Discriminant model to better distinguish the persons with single face sample based on the generic training set, the within-class scatter matrix and the class mean of each person given its single enrolled sample

In [7] M. Yang, L. Zhang, J. Yang and D. Zhang has proposed a Robust sparse coding algorithm in which it eliminates the sparsity-constrained robust regression problem. The RSC enhances the MLE (maximum likelihood estimation) solution of the sparse coding problem, and it is more robust to the corrupted images due to occlusions in outliers.

In [8] M. Aharon, M. Elad and A. Bruckstein has proposed a series of dictionaries. It is an iterative method that alternates between sparse coding based on the current dictionary and a process of updating the dictionary atoms for better recognition purpose.

In [9] M. Yang, L. Van Gool and L. Zhang has proposed sparse variation dictionary learning method by jointly learning a projection to connect the generic training set with the gallery set. so that various variations in face images, including illumination, expression, occlusion, pose, etc., can be better handled.

In[10] M. Yang, L. Zhang, X. Feng and D. Zhang has proposed an algorithm which utilizes the Fisher discrimination Dictionary Learning method by using both the discriminative information from the reconstruction error and sparse coding coefficients.

In [11] C.-P. Wei and Y.-C F. Wang has proposed an optimization algorithm which jointly solves the tasks of auxiliary dictionary learning and sparse-presentation based face recognition.

In [12]J. Wright, A. Y. Yang, A. Ganesh, S. S. Sastry, and Y. Ma, has proposed an algorithm due to varying pixel values by linear regression model to recognize the occluded or disguised part in the corrupted image using sparse matrix.

In [13] W. Deng, J. Hu and J. Guo has proposed an Extended Sparse Representation-Based Classifier (ESRC) that applies an auxiliary intraclass variant dictionary to represent the variation between the training and testing images. If the dictionary and gallery are properly constructed, SRC algorithms can generalize well to the large-scale face recognition problem.

\section{Proposed Work}

The main purpose is to ascertain the establish the question input image $\mathrm{y}$. The gallery $\mathrm{D}$ consists of knowledge sets from $\mathrm{L}$ categories and auxiliary wordbook learning $\mathrm{A}$ is learned from the external data. 


\section{International Journal of Science and Research (IJSR) \\ ISSN (Online): 2319-7064}

Index Copernicus Value (2015): 78.96 | Impact Factor (2015): 6.391
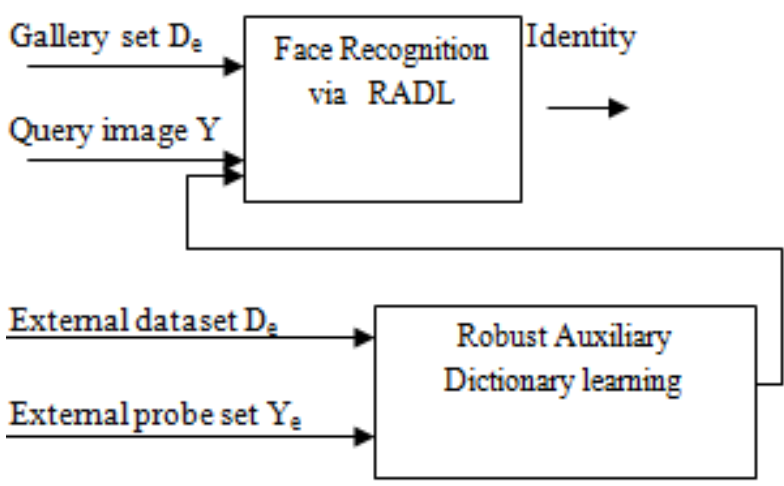

Figure 1: Flow chart for undersampled face recognition

In previous methods residue $(\rho)$ function is introduced for identifying and authentication, but the residue function value has high value for the low and high occlusions which is not suitable for verifying the undersampled data. Our proposed methodology deals with the problems that are occurring frequently due to occlusion and illumination problems.

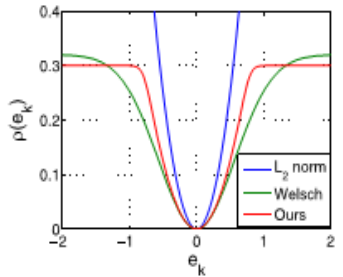

(a)

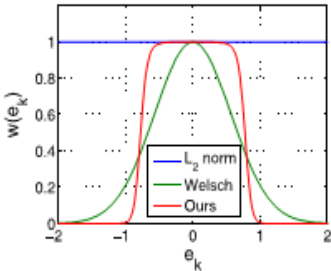

(b)
Figure 2: a) Residue function $(\rho)$ and Weight function(W)

\section{Robust Auxiliary Dictionary Learning (RADL)}

In ESRC [12] based classification residual function( $\rho)$ is applied to identify and discard the corrupted images but in our proposed work methodology weight function $(\omega)$ is introduced[Fig 2] in order to reduce the ambiguity and verifying the image of a person which is suitable for the Undersampled data set. This weight function integrates dictionary learning and classification rule for improved and robust recognition performance. Suppose the external data set contains $\mathrm{p}$ subjects, partition these data into probe set $Y_{e}$ and gallery set $D_{e}$. The probe set consists of $\mathrm{N}$ pictures with totally different category variations of $\mathrm{p}$ subjects and therefore the gallery set contains solely few pictures per subject. To learn an auxiliary dictionary learning for modelling the intra-class image variants is to update the sparse coding by solving the following minimization problem

$$
\begin{gathered}
\min || W_{g}\left(Y_{e}{ }^{i}-\left[D_{e}, A\right] X^{i}\right)\|+\lambda\| X^{i}\|++\eta\| W_{c}\left(Y_{e}{ }^{i}-\right. \\
D_{e} \delta_{i l}\left(X_{d}^{i}\right)-A X_{d}^{i} \|
\end{gathered}
$$

where $\mathrm{W}_{\mathrm{g}}$ and $\mathrm{W}_{\mathrm{c}}$ are the weight functions

from equation 1 the first term indicates the data representation, the second term indicates the sparsity constraint and third term indicates the reconstruction error, and the optimal solution for updating the auxiliary dictionary $\mathrm{A}$ is

$$
\begin{array}{r}
\left(\sum _ { i = 1 } ^ { N } ( ( \breve { W } _ { g } ^ { i } ) ^ { T } \breve { W } _ { g } { } ^ { i } + ( \breve { W } _ { c } { } ^ { i } ) ^ { T } \breve { W } _ { c } { } ^ { i } ) ^ { - 1 } * \left(\sum _ { i = 1 } ^ { N } \left(\left(\breve{W}_{g}^{i}\right)^{T} \Phi_{g}{ }^{i}+\right.\right.\right. \\
\left.(W C i) T \Phi_{C} i\right)
\end{array}
$$

the above equation is obtained by iteratively solving the eq (1) through partial differentiation.

\section{Face Recognition via Robust Auxiliary Dictionary Learning}

In ESRC[12] the given input image is supposed to know the type of occlusion of image $\mathrm{y}$ and present in the dictionary A which is not possible in the real time scenarios. To address this minimization problem like the following

$$
\min \rho\left(y-[D, A]\left[\begin{array}{l}
X_{d} \\
X_{a}
\end{array}\right]\right)+\lambda\|X\|
$$

from the above equation the residue function $(\rho)$ [Fig 2] is not suitable for the undersampled data which is replaced by the weight functions for better recognition in the undersampled data. Therefore the above equation 3 can be replaced by the weight function using $\mathrm{W}$ by applying the existing techniques such as Homoptopy[16], Iterative Shrinkage-Thresholding, or Alternative Direction Method.

The following equation is used to address the minimization problem is

$$
\min \| W\left(y-[D, A]\left[\begin{array}{l}
X_{d} \\
X_{a}
\end{array}\right]\|+\lambda\| X \|\right.
$$

the following is a classification rule to classify y in order to determine in which class the following data is present

$$
l^{*}=\operatorname{argmin} \| W^{*}\left(y-[D, A]\left[\begin{array}{c}
\delta_{l}\left(X_{d}^{*}\right) \\
X_{a}
\end{array}\right] \|\right.
$$

where $X^{*}=\left[X_{d}{ }^{*} X_{a}{ }^{*}\right]$ after solving the above equation $\mathrm{y}$ is assigned to the smallest reconstruction error of the class. The weighted matrix $\mathrm{W}^{*}$ lowers the influence of pixels that are poorly constructed including the corrupted and occluded ones.

\section{Conclusion}

Undersampled Face Recognition is a challenging topic in face recognition where the faces are subjected to the occlusion and disguise which are prone to authentication and authorization. We did a lot of research in this field in order to address the current problems that are facing when undersampled data is present in the database. In this paper, we observed that we are getting a better result by using weight function when compared to residue function in face recognition. Furthermore, this method achieves better recognition, when the image is corrupted. With the help of Robust Auxiliary, Dictionary Learning faces are recognized accurately from the database.

\section{References}

[1] Cha Zhang \& Zhengyou Zhang (2010) "A Survey of Recent Advances in Face Detection", Tech. Rep., MSRTR-2010-66, Microsoft Research, Microsoft Corporation One Microsoft Way, Redmond, WA 98052, http://www.research.microsoft.

[2] T. Ahonen, A. Hadid, and M. Pietikainen, "Face description with local binary patterns: Application to face recognition," IEEE Trans. Pattern Anal. Mach. Intell., vol. 28, no. 12, pp. 2037-2041, Dec. 2006.

[3] Marios Kyperountas, Anastasios Tefas, Ioannis Pitas, "Face recognition via adaptive discriminant clustering" 


\section{International Journal of Science and Research (IJSR) \\ ISSN (Online): 2319-7064 \\ Index Copernicus Value (2015): 78.96 | Impact Factor (2015): 6.391}

Reseach gate International Conference on Image Processing, January 2008.

[4] M. Turk and A. Pentland, "Eigen faces for Recognition", Journal of Cognitive Neuroscience Volume 3, Number 1,1991.

[5] Peter N. Belhumeur, Joao P. Hespanha, and David J. Kriegman, "Eigen faces vs Fisher faces: Recognition using Class Specific Linear Projection" IEEE Transactions on Pattern Analysis and Machine Intelligence archive Volume 19 Issue 7, July 1997.

[6] Y. Su, S. Shan, X. Chen, and W. Gao, "Adaptive generic learning for face recognition from a single sample per person," in Proc. IEEE Conf. Comput. Vis. Pattern Recognit. (CVPR), Jun. 2010, pp. 2699-2706.

[7] M. Yang, L. Zhang, J. Yang, and D. Zhang, "Robust sparse coding for face recognition," in Proc. IEEE Conf. Comput. Vis. Pattern Recognit. (CVPR), Jun. 2011, pp. 625-632.

[8] M. Aharon, M. Elad, and A. Bruckstein, "K-SVD: An algorithm for designing overcomplete dictionaries for sparse representation," IEEE Trans. Signal Process., vol. 54, no. 11, pp. 4311-4322, Nov. 2006.

[9] M. Yang, L. Van Gool, and L. Zhang, "Sparse variation dictionary learning for face recognition with a single training sample per person," in Proc. IEEE Int. Conf. Comput. Vis. (ICCV), Dec. 2013, pp. 689-696.

[10] M. Yang, L. Zhang, X. Feng, and D. Zhang, "Fisher discrimination dictionary learning for sparse representation," in Proc. IEEE Int. Conf. Comput. Vis. (ICCV), Nov. 2011, pp. 543-550.

[11]C.-P. Wei and Y.-C. F. Wang, "Learning auxiliary dictionaries for undersampled face recognition," in Proc. IEEE Int. Conf. Multimedia Expo (ICME), Jul. 2013, pp. 1-6.

[12] J. Wright, A. Y. Yang, A. Ganesh, S. S. Sastry, and Y. Ma, "Robust face recognition via sparse representation," IEEE Trans. Pattern Anal. Mach. Intell., vol. 31, no. 2, pp. 210-227, Feb. 2009.

[13] W. Deng, J. Hu, and J. Guo, "Extended SRC: Undersampled face recognition via intraclass variant dictionary," IEEE Trans. Pattern Anal. Mach. Intell., vol. 34, no. 9, pp. 1864-1870, Sep. 2012.

[14] Stan Z. Li,RuFeng Chu, ShengCai Liao, and Lun Zhang, "Illumination Invariant Face Recognition Using NearInfrared Images" IEEE transactions on pattern analysis and machine intelligence, vol. 29, no. 4, April. 2007.

[15] Ole Helvig Jensen," Implementing the Viola-Jones Face Detection Algorithm", Kongens Lyngby 2008, IMM M.Sc.-2008-93, ISBN 87-643-0008-0, ISSN 1601-233X.

[16] A. Y. Yang, S. S. Sastry, A. Ganesh and Y. Ma,"Fast 11-minimization algorithms and an application in robust face recognition: A review," in proc. $17^{\text {th }}$ IEEE Int. Conf. Image Process.(ICIP), Sep 2010, pp.1849-1852.

[17] W. Zhao, R. Chellappa, A. Krishnaswamy, "Discriminant analysis of Principal Components for Face Recognition", proc. Third IEEE Int. Conf. on the Automatic face and Gesture Recognition, 1998. 